\title{
Direct generation of optical vortices
}

\author{
Mathew D. Williams, Matt M. Coles, David S. Bradshaw, and David L. Andrews* \\ School of Chemistry, University of East Anglia, Norwich NR4 7TJ, United Kingdom
}

(Received 20 December 2013; published 21 March 2014)

\begin{abstract}
A detailed scheme is established for the direct generation of optical vortices, signifying light endowed with orbital angular momentum. In contrast to common techniques based on the tailored conversion of the wave front in a conventional beam, this method provides for the direct spontaneous emission of photons with the requisite field structure. This form of optical emission results directly from the electronic relaxation of a delocalized exciton state that is supported by a ringlike array of three or more nanoscale chromophores. An analysis of the conditions leads to a general formulation revealing a requirement for the array structure to adhere to one of a restricted set of permissible symmetry groups. It is shown that the coupling between chromophores within each array leads to an energy level splitting of the exciton structure, thus providing for a specific linking of exciton phase and emission wavelength. For emission, arrays conforming to one of the given point-group families' doubly degenerate excitons exhibit the specific phase characteristics necessary to support vortex emission. The highest order of exciton symmetry, corresponding to the maximum magnitude of electronic orbital angular momentum supported by the ring, provides for the most favored emission. The phase properties of the emission produced by the relaxation of such excitons are exhibited on plots which reveal the azimuthal phase progression around the ring, consistent with vortex emission. It is proven that emission of this kind produces electromagnetic fields that map with complete fidelity onto the phase structure of a Laguerre-Gaussian optical mode with the corresponding topological charge. The prospect of direct generation paves the way for practicable devices that need no longer rely on the modification of a conventional laser beam by a secondary optical element. Moreover, these principles hold promise for the development of a vortex laser, also based on nanoscale exciton decay, enabling the production of coherent radiation with a tailor-made helical wave front.
\end{abstract}

DOI: 10.1103/PhysRevA.89.033837

PACS number(s): 42.50.Tx, 03.70.+k, 42.50.Ct, 81.07.Nb

\section{INTRODUCTION}

In recent years, the field of optics has been enriched by the rapid development of theory and experiments involving light endowed with orbital angular momentum (OAM), distinct and separable from any spin angular momentum associated with the polarization state [1]. The theoretical basis for the propagation of such beams, which are distinguished by an azimuthal progression of phase around an axis of field singularity, was first established in a series of works introducing the associated concept of an "optical vortex" [2-5]. The classical representation is a bundle of rays whose wave vector precesses about the axis of beam propagation. The phase discontinuity at the core is responsible for field distributions whose transverse structures comprise one or more rings, centered upon the core. Recent advances have consolidated the theory and allowed the quantum nature of such beams to be fully elicited.

The detailed structure of vortex beams is primarily characterized by a topological charge, $l$ (signifying an OAM of it per photon), which can take any real integer value, a positive or negative parity denoting either left- or right-handed gyration, respectively. Further aspects are determined by the transverse form of the electromagnetic fields, for which a Laguerre-Gaussian radial distribution is the most commonly assumed. A beam with the topological charge $l$ thus exhibits a field distribution in the form of $l$ intertwined helices, each of which completes a turn of $2 \pi$ radians about the axis over a span of $l$ wavelengths, as exemplified in Fig. 1 [6-8].

\footnotetext{
*Author to whom correspondence should be addressed: d.1.andrews@uea.ac.uk
}

There is an increasing recognition of the wide scope to exploit the unique properties of optical vortex beams, with wide ranging applications [9-11]. One of the key motivators in current experimental pursuits is the aim of maximizing optical data transfer speeds, based on the information content per photon-although due consideration as to how much information can ultimately be conveyed in a single quantum of light is required [12-18]. In this connection, it is notable that schemes for separating and identifying the OAM content of vortex light are rapidly achieving higher levels of fidelity, primarily through angular sorting [19-22].

The particular aspiration for achieving increased speed and data content of free-space data transmission [23,24] is, however, potentially compromised by atmospheric turbulence [25-28]. Another proven application is in optical manipulation [29-33], where the capacity to exert a far greater torque than a regular circularly polarized beam has earned the technique the name "optical spanners (wrench)" [34-37]. There are also numerous methods utilizing structured light in imaging applications, edge contrast enhancement [38] and microparticle trapping [39], for example.

Up until recently [40] it was deemed only possible to produce a vortex beam by imparting OAM onto a preexisting beam, through wave-front modification. There are several well documented and experimentally proven mechanisms for bestowing OAM character onto a conventional beam, for example, by passage through pitchfork holograms [41-43], paired cylindrical lenses [44], q plates [45], hyperbolic metamaterials [46], spiral phase plates [47], or spatial light modulators (SLMs) [48,49]. The many recent advances in such devices have provided new insights into the interaction and coupling between the spin and orbital angular 


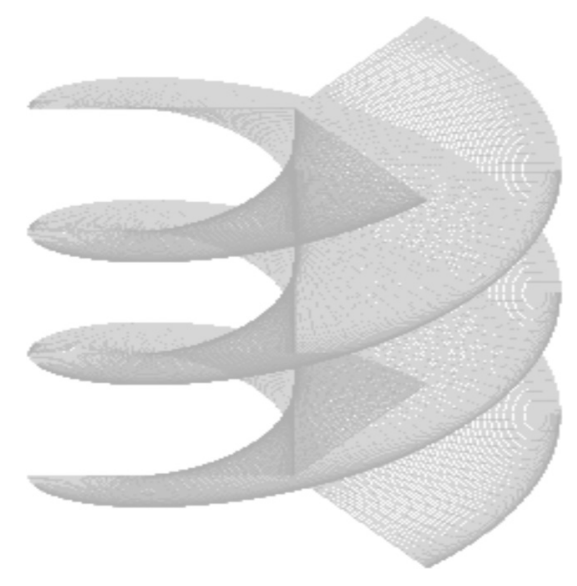

FIG. 1. Schematic representation of the wave front for a typical $l=3$ optical vortex, over the span of three wavelengths. The interval between successive wave fronts is one wavelength.

momenta [50], experimentally exploited in a $q$ plate, and also the nonuniform polarization fields of other "vector" beams [51-53]. A common, significant disadvantage of most previous devices is that they can deliver output varying in not only the integer of topological charge but also the sign of the emission.

By way of contrast, a method for the direct generation of a vortex beam, based on optical emission from a nanoscale array, is now fully investigated [54,55]. First, we examine the physical principles for photon emission arising from the relaxation of an excited array system with a geometry defined in the following. The aim of this analysis is to ascertain the key symmetry features of the locally extended electronic excitation from such an array that generates the vortex photon. The theoretical foundation for the emission mechanism is examined in detail, before explicit demonstration and characterization of the wide ranging outputs that are achievable. To conclude we identify possible future developments, including the prospect of an OAM laser.

\section{PRINCIPLES OF PHOTON EMISSION FROM A MOLECULAR ARRAY}

Symmetry constraints on the emitter array are governed by certain fundamental principles. Both for simplicity and practicality — and in order to establish a directionality for the emitted light - it is expedient to assume a ringlike array in which the centers of each component lie in a single plane. The primary consideration is then to establish a correlation between the symmetry properties of the sought emission, and those of the exciton state from which the vortex beam is to be launched. In an array of essentially planar form a progression of phase around the ring can deliver the required effect, operating in a fashion similar to a helically ramped structure such as a phase plate-without the radial discontinuity at the edge of the latter optical element. While this phase progression is an obvious geometric corollary of a propagating vortex beam, it is also a structural feature that can be transcribed from a static material component.

Since we are concerned with emission, rather than transmission, the sought phase property is determined by the character of each specific excited state, rather than the array upon which that state resides. In turn this means that, given an array with a sufficient number of components, it is possible for vortex structures of various topological charge to be generated from a single array. The extended, partially delocalized excitation of essentially ringlike form will have a symmetry that is lower than that of the material system upon which it is based. To achieve such a form of excitation is impossible with the atomic components of any conventional source: Since nonspherical emitters are required, molecular sources are an obvious choice. Crucially, neither the individual emitters nor the array need have a chiral form. Similar principles are deployed in connection with the plasmonic structures known as planar chiral metamaterials [56-60].

Suitable structures for the arrays can be found by examining the Schoenflies point-group tables $[61,62]$. We require pointgroup symmetries that feature biaxial degeneracy (for the two axes defining the plane of the ring), yet lack "vertical" mirror symmetry (whose presence would undermine the sought progression of phase around the ring). Satisfying these criteria, suitable families of groups prove to be $C_{n}, C_{n h}, S_{n}$, $T, T_{h}$, where $n$ is the number of chromophores comprising the ring. Within these groups, attention now focuses on the doubly degenerate irreducible representations, which are the bases for the requisite forms of delocalized electronic exciton. Our concern is with the transitions that occur as those exciton states decay radiatively [63]. Assuming that such decay terminates in a ground state belonging to the totally symmetric representation, then the symmetry character of the initial excited state maps directly onto the symmetry of the decay transition, and hence the vortex structure of the emitted electromagnetic radiation. (Formally, the direct product of representations for photon electronic field, and that of the excitonic state, has to include the totally symmetric representation.) Table I details the possible integer values of orbital angular momentum that can be emitted from an array with symmetry defined below, characterized by its respective Schoenflies point group.

TABLE I. Summary of the allowed topological charge $l$, for OAM outputs based on arrays of the allowed symmetry groups. For the $S_{n}$ groups, $q=0$ if $i \notin\left\{S_{n}\right\} ; q=1$ if $i \in\left\{S_{n}\right\}$. In addition (but not shown), the groups $T$ and $T_{h}$ may also support a unit topological charge. The entries in the last row, for the general case, express the necessary conditions incorporating a floor function [64].

\begin{tabular}{lcc}
\hline \hline & \multicolumn{2}{c}{ Symmetry group } \\
\cline { 2 - 3 } Number of emitters & $C_{n}, C_{n h}$ & $S_{n}$ \\
\hline 3 & 1 & - \\
4 & 1 & - \\
5 & 1,2 & 1 \\
6 & 1,2 & - \\
7 & $1,2,3$ & $1,2,3$ \\
8 & $1,2,3$ & - \\
9 & $1,2,3,4$ & 1,2 \\
10 & $1,2,3,4$ & - \\
11 & $1,2,3,4,5$ & $1,2,3,4,5$ \\
12 & $1,2,3,4,5$ & $|l| \leqslant\left\lfloor\left(\frac{1}{2}\right)^{q} \frac{n-2}{2}\right\rfloor$ \\
$n$
\end{tabular}




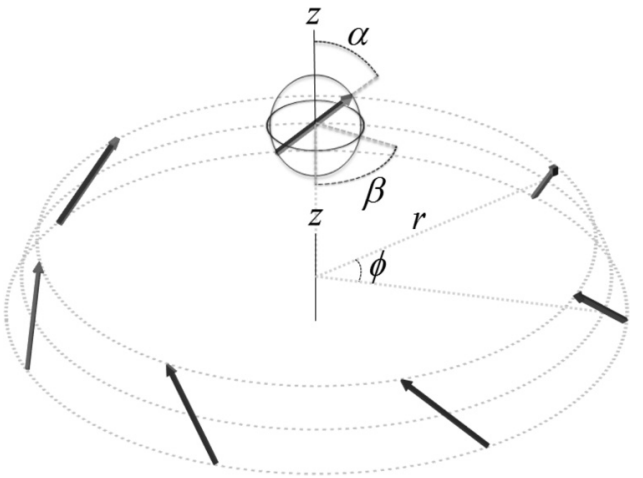

FIG. 2. Schematic depiction of the chromophore array, illustrating the case of seven emitters, in a plane whose normal is the $z$ direction. The dotted lines are guides for the eye. The angle $\Phi$ designates the azimuthal position in the plane; $\alpha$ and $\beta$ are angles that specify the identical local orientation of each emitter.

By setting the angle $\alpha=\pi / 2$ in Fig. 2, the array will move to a higher symmetry group $C_{7 h}$ from $C_{7}$, by virtue of the additional horizontal mirror symmetry. The same applies for point groups of arbitrary rotational symmetry. For similar reasons it is necessary that $\alpha \neq 0$, nor $\beta=0$ or $\pi$, to forestall the introduction of additional symmetry that undermines the sought chirality.

\section{EXCITON HAMILTONIAN AND WAVE FUNCTIONS}

Having considered the symmetry requirements, we now turn to the detailed form of the delocalized electronic wave functions and the associated energies. Given $n$ emitters, it is immediately evident that a state in which only one chromophore is electronically excited, and the others are in their ground state, does not represent a stationary state of the system due to the coupling between adjacent emitters. In order to determine the stationary states, a block diagonalized form of the array Hamiltonian is required, with the singly excited states as the basis. The stationary states then emerge in the form of superpositions of these basis states with normalized coefficients [65].

In general the array Hamiltonian may be expressed in matrix form as follows: ${ }^{1}$

$$
H_{r s}=E_{u} \delta_{r s}+\left[\boldsymbol{\mu}_{r} \cdot \mathbf{V}\left(\mathbf{R}_{r s}\right) \cdot \boldsymbol{\mu}_{s}\right]\left(\delta_{r-1, s(\bmod n)}+\delta_{r(\bmod n), s-1}\right),
$$

where each element of the $n$-square matrix relates to a pair of emitters $\{r, s\} \in \mathbb{N}$. Each diagonal element $E_{u}$ signifies the energy of an isolated nanoemitter in its excited electronic level $u$; the off-diagonal elements denote interactions between emitter pairs. These interactions, in turn, are determined by the electrodynamic coupling $\mathbf{V}\left(\mathbf{R}_{r s}\right)$ between neighboring transition dipole moments $\boldsymbol{\mu}_{r}$ and $\boldsymbol{\mu}_{s}$-all of which are identical in magnitude to the coupling between a nominated

\footnotetext{
${ }^{1}$ This formula correctly generates additional off-diagonal terms missing from a simplified formula given previously [40].
}

pair such as nanoemitters 1 and 2:

$$
\mathbf{V}\left(\mathbf{R}_{r s}\right) \equiv V_{r,(r+1) \bmod n}\left(k_{u}, \mathbf{R}_{r,(r+1) \bmod n}\right) \equiv V_{12}\left(k_{u}, \mathbf{R}_{12}\right) .
$$

Here, $\mathbf{R}_{r s}$ is the vector displacement between the relevant nanoemitters [66]: $R$ is defined by $\mathbf{R}_{r}-\mathbf{R}_{s} \equiv \mathbf{R}_{r s}=R \hat{\mathbf{R}}_{r s}$, the caret here denoting a unit vector. Under the symmetry conditions described in the previous section, all the nonzero off-diagonal elements of Eq. (1) return the same scalar value, $U$. Explicitly, the latter is expressed as

$$
\begin{aligned}
U= & \boldsymbol{\mu}_{r} \cdot \mathbf{V}\left(\mathbf{R}_{r s}\right) \cdot \boldsymbol{\mu}_{s} \\
\equiv & \frac{e^{i k_{u} R}}{4 \pi \varepsilon_{0} R^{3}}\left[\left\{1-i k_{u} R-\left(k_{u} R\right)^{2}\right\}\left(\boldsymbol{\mu}_{1}^{0 u} \cdot \boldsymbol{\mu}_{2}^{0 u}\right)\right. \\
& \left.-\left\{3-3 i k_{u} R-\left(k_{u} R\right)^{2}\right\}\left\{\left(\boldsymbol{\mu}_{1}^{0 u} \cdot \hat{\mathbf{R}}_{12}\right)\left(\boldsymbol{\mu}_{2}^{0 u} \cdot \hat{\mathbf{R}}_{12}\right)\right\}\right],
\end{aligned}
$$

where the individual electric dipole transition moments are defined by $\boldsymbol{\mu}_{r}^{0 u} \equiv\left\langle\xi^{r ; 0}\left|\boldsymbol{\mu}^{(r)}\right| \xi^{r ; u}\right\rangle$ and $k_{u}=E_{u} / \hbar c$.

To diagonalize Eq. (1), and identify the eigenstates corresponding to the excited-state splitting (as detailed in Sec. IV), we require that

$$
\begin{aligned}
0 & =\operatorname{det}\left[H_{r s}-\lambda I\right] \\
& =\operatorname{det}\left[\left(E_{u}-\lambda\right) \delta_{r s}+V\left(\delta_{r-1, s(\bmod n)}+\delta_{r(\bmod n), s-1}\right)\right] .
\end{aligned}
$$

Moreover, the result for the $n$ eigenfunction excitons is secured as follows:

$\left|\psi_{p}\right\rangle=\frac{1}{\sqrt{n}} \sum_{r=1}^{n} \varepsilon_{n}^{(r-1) p}\left|\xi^{r ; u}\right\rangle \prod_{s \neq r}\left|\xi^{s ; 0}\right\rangle, \quad p \in\{1, \ldots, n\}$.

In this linear combination, $\left|\xi^{r ; u}\right\rangle$ is a state function corresponding to an emitter $r$ in electronic state $u$, and $\varepsilon_{n}=$ $\exp (2 \pi i / n)$. In every summand of Eq. (5), one chromophore is in the electronically excited state $u$, while the others are in their ground states. The energy eigenvalues associated with the above exciton states are generally expressible in the form

$$
E_{p}=E_{u}+2 U \cos (2 p q / n)
$$

with $-\lfloor(n-1) / 2\rfloor<q \leqslant\lfloor n / 2\rfloor$ and with this index $q$ related to $p$ in Eq. (5) through

$$
q=\left\{\begin{array}{l}
p \mid p \leqslant\lfloor n / 2\rfloor \\
p-n \mid p>\lfloor n / 2\rfloor
\end{array} .\right.
$$

It is also possible for two emitters to be simultaneously excited within a given exciton ring, although this will not be considered any further. Despite the corresponding analysis having a broadly similar basis in theory, the achievement of such a configuration would be experimentally much more demanding, since the initial excitation would necessitate the absorption of two quanta within the excited-state lifetime and, accordingly, a significantly higher intensity for the excitation beam.

Table II, which summarizes the relationships between the indexes $p, q$ for a range of $n$ values, $(3 \leqslant n \leqslant 7)$, also indicates the irreducible representation of the corresponding family of point groups $C_{n}$, associated with each excitonic state. Their properties are described in the following section. 
TABLE II. List of the irreducible representations (irreps) of the $C_{n}$ exciton states for $n=3-7$.

\begin{tabular}{ccccccccc}
\hline \hline$n$ & $p$ & 1 & 2 & 3 & 4 & 5 & 6 & 7 \\
\hline 3 & $q$ & 1 & -1 & 0 & & & & \\
& Irrep & $E_{1}$ & $E_{1}$ & $A$ & & & & \\
4 & $q$ & 1 & 2 & -1 & 0 & & & \\
& Irrep & $E_{1}$ & $B$ & $E_{1}$ & $A$ & & & \\
5 & $q$ & 1 & 2 & -2 & -1 & 0 & & \\
& Irrep & $E_{1}$ & $E_{2}$ & $E_{2}$ & $E_{1}$ & $A$ & & \\
6 & $q$ & 1 & 2 & 3 & -2 & -1 & 0 & \\
& Irrep & $E_{1}$ & $E_{2}$ & $B$ & $E_{2}$ & $E_{1}$ & $A$ & \\
7 & $q$ & 1 & 2 & 3 & -3 & -2 & -1 & 0 \\
& Irrep & $E_{1}$ & $E_{2}$ & $E_{3}$ & $E_{3}$ & $E_{2}$ & $E_{1}$ & $A$ \\
\hline \hline
\end{tabular}

\section{STRUCTURE OF THE EXCITONIC ENERGY LEVELS}

Due to their differences in symmetry, the exciton eigenstates will also exhibit differences in energy, manifest as line splittings centered upon the frequency of an isolated emitter. On examination of an exemplary nanoarray with $n=3$ emitters, we determine that the positioning of exciton levels leads to one nondegenerate state (belonging to the totally symmetric representation $A$ ), with energy $E_{u}-2 U$, and two doubly degenerate ( $E$ representation) states of energy $E_{u}+U$. Furthermore, the sign of the coupling $U$ is readily shown to be positive for all $n \geqslant 3$, which is a requirement to form a singularity along the axis of propagation. The $E$ excitons exist in the form of pairs, one with a left-handed and the other a right-handed sense of phase progression around the ring. These states exactly correspond with the sought distributions of phase about the symmetry axis, and are separated in energy from the $A$ form by $3 U$, a principle that should enable the selective excitation of one symmetry type [65]. As will emerge, the $E$ exciton distributions accordingly map onto the optical field produced by their decay, leading to an optical vortex.

The different symmetry designations of excitons with representations $E_{q}, A$ (and also $B$, if present) are reflected in their association with different energies. The decay of each doubly degenerate exciton labeled $q$ can therefore result in the emission of a photon with a characteristic wavelength, corresponding to the initial excitation level. In practice, consistent with Kasha's rule (which applies in the context of vibrational sublevels), the emission usually relates to the energy gap between the lowest-lying energy level and the ground state, as illustrated by Fig. 3. In most physical cases, the exciton pair with the lowest energy will be associated with the highest values of $|q|$ and, as it emerges, the largest topological charge for the emitted vortex.

\section{OPTICAL VORTEX EMISSION FROM A CIRCULAR ARRAY}

To determine the phase map of the emitted beam, which corresponds to the described excitons, an expression for the electric field $\mathbf{E}_{p}\left(\mathbf{R}_{D}\right)$ from each constituent nanoemitter is

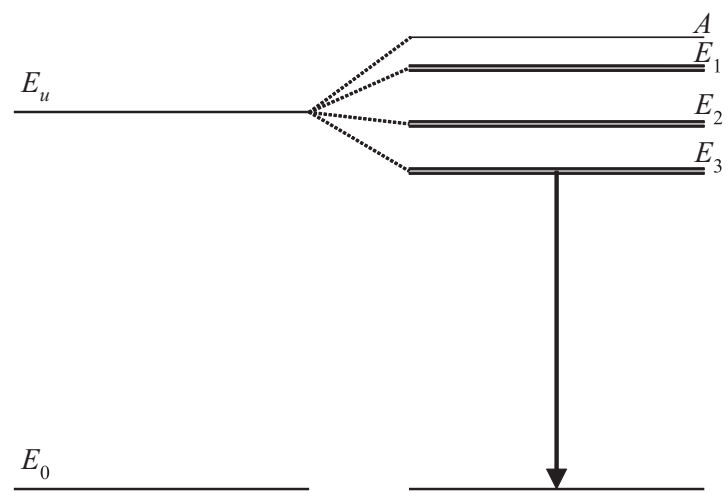

FIG. 3. Excitonic irreducible representations and Davydov energy level splitting for an array of $C_{7}$ point-group symmetry.

required; i.e.,

$$
\begin{aligned}
\mathbf{E}_{p}\left(\mathbf{R}_{D}\right)= & \sum_{r}^{n} \frac{e^{i k R_{D r}} \varepsilon_{n}^{(r-1) p}}{4 \pi \varepsilon_{0} R_{D r}^{3}}\left\{\left[\left(\hat{\mathbf{R}}_{D r} \times \boldsymbol{\mu}_{r}^{0 u}\right) \times \hat{\mathbf{R}}_{D r}\right] k^{2} R_{D r}^{2}\right. \\
& \left.+\left[3 \hat{\mathbf{R}}_{D r}\left(\hat{\mathbf{R}}_{D r} \cdot \boldsymbol{\mu}_{r}^{0 u}\right)-\boldsymbol{\mu}_{r}^{0 u}\right]\left(1-i k R_{D r}\right)\right\},
\end{aligned}
$$

where $\mathbf{R}_{D}$ signifies the displacement, relative to the ring center, of a point of measurement or detection. Notably, each term in Eq. (8) carries the phase factor, $\varepsilon_{n}^{(r-1) p}$, from the corresponding emitter component in Eq. (5), thus delivering the sought progression in phase around the ring. At any point in space, the most appropriate measure of the phase for the emitted radiation is the function defined by

$$
\theta_{i}(\mathbf{R})=\arg \left\{\mathbf{E}_{p ; i}\left(\mathbf{R}_{D}\right)\right\} .
$$

Here the phase $\theta$ is given as the principle argument of the complex electric field vector. Our analysis thus enables the identification of expressions for the phase $\theta$ relevant for each of the electric field components in the $x, y$, and $z$ directions individually, where $i$ indexes the Cartesian components. Typical maps of the electromagnetic phase distributions, shown in Figs. 4-6, exhibit the variation of the phase in planes parallel to the source array, for several combinations of chromophore number, exciton symmetry, and distance from the source. The plot at the top left of Fig. 4 serves to reinforce the conclusion that two emitters cannot generate the required field chirality; the remaining panes in this figure show the $l=1$ vortex structures supported by rings with three to six chromophore components. Figure 5 displays the changes that result from different $l$ values, and varying distance from the source plane; Fig. 6 enables identification of the phase properties in individual polarization components, for one representative case.

The phase properties of the electromagnetic fields emitted by the arrays in each of these cases can be shown to map exactly to the azimuthal phase dependence of the corresponding Laguerre-Gaussian optical mode. Specifically, it transpires that for any field with an $l$ value that is a prime number, its projection onto a mode with any other value of the topological charge in the interval $[-l, l]$ delivers a vanishing integrated result. 

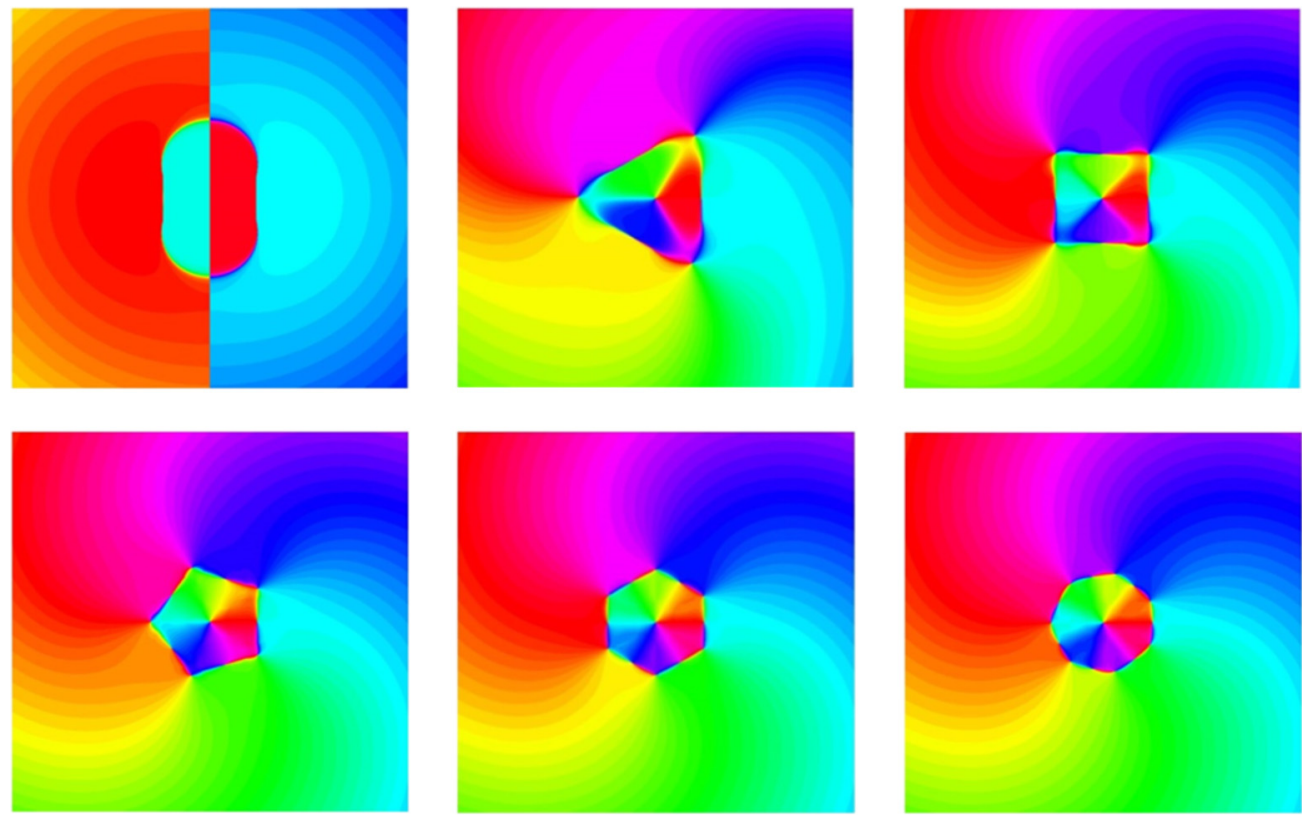

FIG. 4. (Color online) Exciton phase cross section of the scalar optical field in the array plane, calculated for a series of arrays supporting optical vortex emission, with an increasing number of chromophores, $n=\{2,3,4,5,6\}, l=-1$, oriented to conform to the $C_{n}$ family of point groups. Each color represents a different phase and the contours radiating in a spiral manner from the ring of emitters are lines of constant phase. The phase cycles through $2 \pi^{\mathrm{c}}$ around concentric circles of constant radius from the ring center. The angle $\alpha$ between the normal to the plane, and the radial position vector of the corresponding emitter, equals $\pi / 4$, as does the angle $\beta$ subtended by each emission dipole moment onto the plane containing the array centers (see Fig. 2). In this case the coupling constant $U$ is positive. Notice the field singularity at the core. In these plots the emitters are radially disposed at a distance of $\lambda / 200 \pi$ from the center, where $\lambda$ is the optical wavelength of emission, and the lateral span across each plot corresponds to distances $R=\lambda / 10 \pi$.
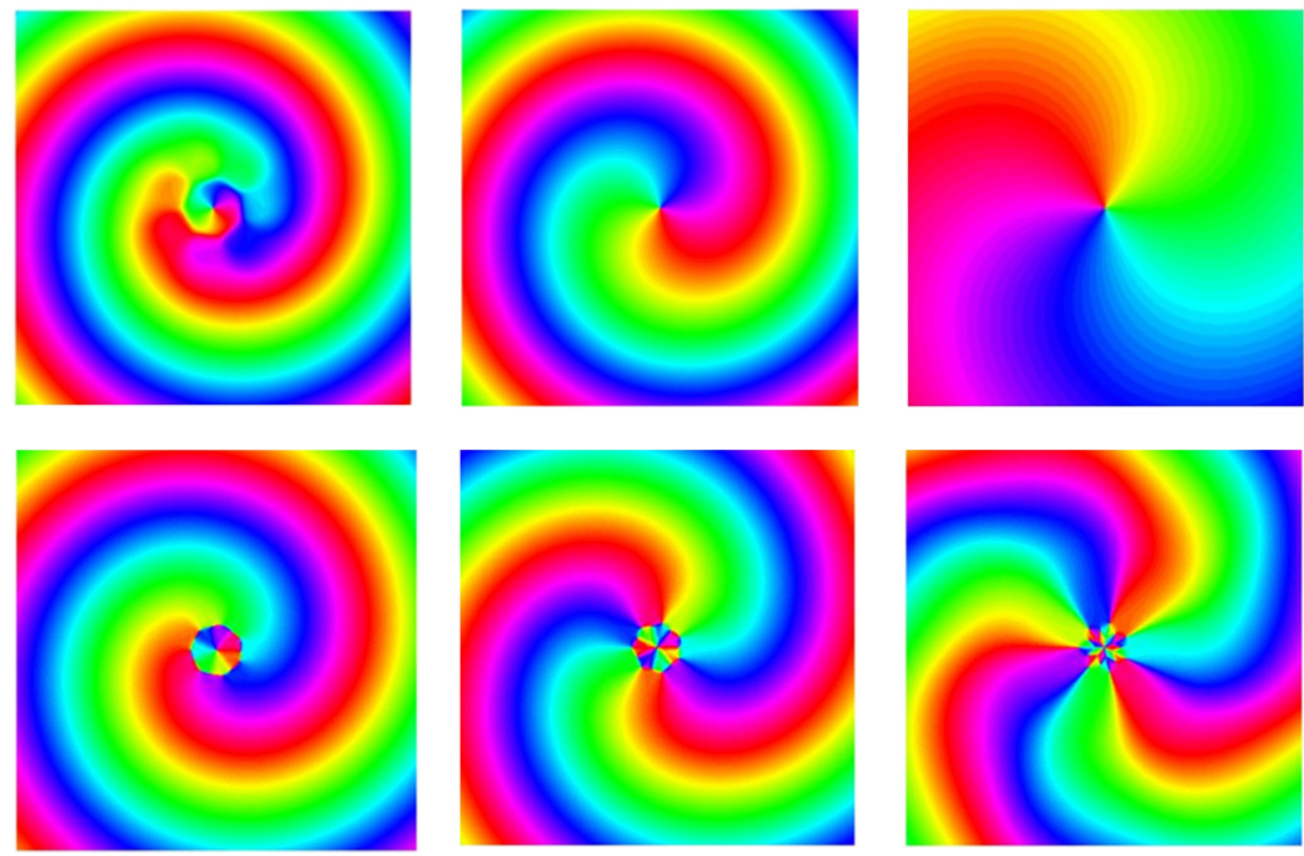

FIG. 5. (Color online) Extending from lowermost right image in Fig. 4, all simulations in this panel have $n=7$, the top row having $l=+1$ fixed; however, the phase progression has been captured incrementally above the array plane, specifically at $z=\{3,24,100000\} \times 10^{-3}$. Now the lines of constant phase on the cross sections can be connected into a plane of constant phase, which is commonly depicted as seen in Fig. 1, with $l=3$ corresponding to that displayed in the lower right corner. The bottom row of the panel displays all possible $|l|$ values plotted from an array of $n=7$; note that all of the negative counterparts are also permissible in addition to the emission of an $l=0$. The physical dimensions of the emitter core are the same as in Fig. 4. 

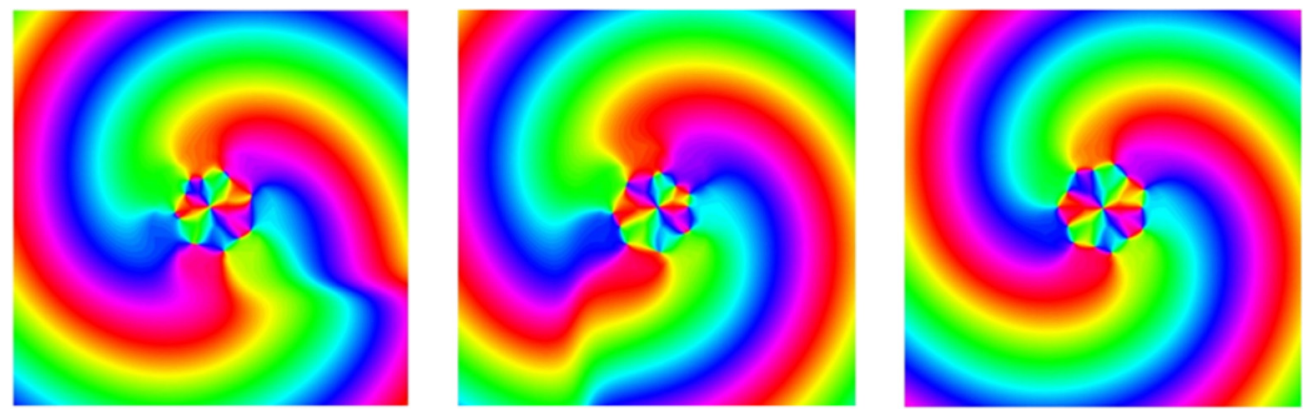

FIG. 6. (Color online) Sagittal, coronal, and transverse planar cross sections intersecting at the ring's center, corresponding to the $E_{x}, E_{y}, E_{z}$, respectively, for an array with $n=7, l=-2$. Physical dimensions of the emitter core as in Fig. 4.

\section{CONSIDERATION OF VORTEX LASER EMISSION BASED ON ARRAY EMISSION}

Since an exciton may decay to produce a photon with vortex beam characteristics, it is speculated that achieving population inversion within a set of such nanoarrays might enable stimulated emission to occur-and hence, with suitably configured optics, provide the mechanism for a vortex laser. Such a device could, for example, be based upon nanofabricated surfaces suitably tailored with preformed, surface-deposited molecular arrays, akin to chiral sculptured thin films [67-69]. The arrays could themselves provide repeat units for a metastructured material as, for example, might comprise stacks within the cavities of a porous silicate, zeolite, or similar lattice. In contrast to other recent propositions for lasers delivering photons endowed with topological charge, this design does not require the use of efficiency-reducing optical elements [70]. It can be expected that vortex laser beams will display subsidiary sidebands and other features associated with vortex instability, as demonstrated recently in studies with Raman-active crystals [71]. Achieving suitable phase matching along the emission direction would undoubtedly be paramount. With sufficiently strong pumping and an optical cavity suitably constructed to support emission in the predetermined direction, normal to the array planes, bulk materials of this kind might then lead to a viable optical vortex laser source.

In further developing and refining the analytical representation of such a device it is anticipated that the embedding medium will have a strong influence on the emission process. This will indeed provide a technical basis for optimizing emission characteristics. It is already known how to create the necessary adaptations to the formulation we have provided; the dipole-dipole interaction tensor in the above calculations may be replaced with the medium-modified form introduced by Juzeliūnas [72]. Experimentally, the feasibility of such a setup also requires additional consideration, with the stability of higher $l$-value states having been questioned in recent work on the decay of high-order vortex modes into groups of more stable unit vortices [73-75]. Such problems have nonetheless been shown to be surmountable: The propagation of OAM modes in a single mode fiber can preserve OAM structure [76].

\section{DISCUSSION}

The present work affords an unusual opportunity to consider the emission of structured light upwards from the single photon level. It is interesting to note the exploitation of a principle that, even though the individual emission dipoles do not exhibit the nature associated with a higher multipole, the array as a whole is capable of producing a field distribution of complex multipolar form. In this way it is possible to overcome the fundamental obstacle that isolated multipole emitters cannot produce radiation imprinted with a corresponding orbital angular momentum. To generate light with OAM it is neither possible nor necessary to invoke an electronic transition of the cognate character in individual emitters $[77,78]$.

Although attention has already been drawn to the more conventional methods of optical vortex generation, it is of interest to note that the presently proposed, fabrication-based method for the generation of structured light represents a very different approach to another recently described computational method, for securing beams shaped in three dimensions [79]—although in the latter case there seems to have been no suggestion that such methods could produce the phase structures associated with optical vortices. Again, although similar concepts have drawn attention to the possibility of producing multivortex emission, from larger antenna structures formed in lattices, the emission wavelength in such cases is determined by geometry, rather than atomic or molecular constitution [80,81]. The latter work is closer in concept to the radio arrays pioneered by Tamburini and co-workers [82]. Again, a key difference in our work is that the material itself, in consequence of its intrinsic molecular electronic states, determines the wavelength of emission.

The analysis elaborated in previous sections represents a combination of principles that are directly amenable to implementation in suitably fabricated molecular arrays. Prior to this, we have purposely endeavored to avoid being too prescriptive concerning the detailed systems of choice. In fact, by extension of this groundwork, a wider variety of possible means of implementation might be envisaged. One obvious alternative to an array of individual molecular components is a multichromophore architecture, which could satisfy the necessary conditions if distinct electronic transitions occur in each of the constituent chromophores [83,84]; such possibilities could offer means to achieve the symmetry exhibited by the structure displayed in Fig. 2. By more extensive means [85,86], bespoke arrays could be directly manufactured to provide for pairwise coupling between neighboring nanoscale antennae. Here, there are options for a variety of planar deposition techniques $[87,88]$, including those that can produce layered chiral thin films of dielectric materials [89], or others that 
provide for tailored metallic structures exhibiting delocalized electronic motions [90,91]. Electron beam and other forms of lithography could be used to etch the surface of a suitable substrate [92-94]. Further opportunities might be afforded by quantum dot nanoarrays [95], especially given the possibility of forming anisotropic components with directional emission properties [96]. Lastly, since magnetic guidance proves amenable to maneuvering magnetizable nanoparticles, this too might afford a means of fulfilling the necessary symmetry conditions without the demands of intrinsic symmetry [97].

In quantum information transfer and processing, the use and miniaturization of vortex light sources is a highly topical field of research [98,99]. Against this context it is notable that our array basis for emission, given consideration of time reversal, clearly offers additional scope for use as a detector of orbital angular momentum. However, the basic physics may be more clouded by the difficulties of quantum measurement, in which the nature of the detector and detection process are inextricably bound. Other methods for detecting orbital angular momentum, based on spatial separation are also already attracting considerable attention [19,20,27]. In forthcoming work a thorough investigation will focus on this aspect of the work. It is also planned to extend current work to include the explicit consideration of superimposing differently centered vortices.

\section{ACKNOWLEDGMENTS}

We gratefully acknowledge funding of this research by the UK Engineering and Physical Sciences Research Council, the Leverhulme Trust, and the University of East Anglia.
[1] I. Bialynicki-Birula and Z. Bialynicka-Birula, J. Opt. 13, 064014 (2011).

[2] J. Nye and M. Berry, Proc. R. Soc. London, Ser. A 336, 165 (1974).

[3] P. Coullet, L. Gil, and F. Rocca, Opt. Commun. 73, 403 (1989).

[4] B. E. A. Saleh and M. C. Teich, Fundamentals of Photonics (Wiley, New York, 1991), p. 104.

[5] L. Allen, M. W. Beijersbergen, R. J. C. Spreeuw, and J. P. Woerdman, Phys. Rev. A 45, 8185 (1992).

[6] M. J. Padgett and J. Courtial, Opt. Lett. 24, 430 (1999).

[7] L. Allen, J. Opt. B: Quantum Semiclassical Opt. 4, S1 (2002).

[8] G. Milione, H. I. Sztul, D. A. Nolan, and R. R. Alfano, Phys. Rev. Lett. 107, 053601 (2011).

[9] L. Allen, S. M. Barnett, and M. J. Padgett, Optical Angular Momentum (Taylor \& Francis Group, Bristol, 2003).

[10] D. L. Andrews, Structured Light and its Applications: An Introduction to Phase-Structured Beams and Nanoscale Optical Forces (Academic, Amsterdam, Boston, 2008), p. 2.

[11] D. L. Andrews and M. Babiker, The Angular Momentum of Light (Cambridge University Press, Cambridge, UK, 2013).

[12] J. Leach, M. J. Padgett, S. M. Barnett, S. Franke-Arnold, and J. Courtial, Phys. Rev. Lett. 88, 257901 (2002).

[13] G. Gibson, J. Courtial, M. Padgett, M. Vasnetsov, V. Pas'ko, S. Barnett, and S. Franke-Arnold, Opt. Express 12, 5448 (2004).

[14] J. C. García-Escartín and P. Chamorro-Posada, Phys. Rev. A 78, 062320 (2008).

[15] C. J. Broadbent, P. Zerom, H. Shin, J. C. Howell, and R. W. Boyd, Phys. Rev. A 79, 033802 (2009).

[16] D. L. Andrews, Proc. SPIE 7754, 77540T (2010).

[17] D. L. Andrews, Proc. SPIE 7950, 79500L (2011).

[18] E. J. Galvez, L. E. Coyle, E. Johnson, and B. J. Reschovsky, New J. Phys. 13, 053017 (2011).

[19] M. N. O’Sullivan, M. Mirhosseini, M. Malik, and R. W. Boyd, Opt. Express 20, 24444 (2012).

[20] M. P. Lavery, D. Roberston, M. Malik, B. Robenburg, J. Courtial, R. W. Boyd, and M. J. Padgett, Proc. SPIE 8542, 85421R (2012).

[21] A.-P. Liu, X. Xiong, X.-F. Ren, Y.-J. Cai, G.-H. Rui, Q.-W. Zhan, G.-C. Guo, and G.-P. Guo, Sci. Rep. 3, 2402 (2013).

[22] A. Dudley, C. Schulze, I. Litvin, M. Duparré, and A. Forbes, Proc. SPIE 8810, 88100E (2013).
[23] G. A. Tyler and R. W. Boyd, Opt. Lett. 34, 142 (2009).

[24] J. Wang, J.-Y. Yang, I. M. Fazal, N. Ahmed, Y. Yan, H. Huang, Y. Ren, Y. Yue, S. Dolinar, M. Tur, and A. E. Willner, Nat. Photonics 6, 488 (2012).

[25] Y. Gu, J. Opt. Soc. Am. A 30, 708 (2013).

[26] Y. Ren, H. Huang, G. Xie, N. Ahmed, Y. Yan, B. I. Erkmen, N. Chandrasekaran, M. P. J. Lavery, N. K. Steinhoff, M. Tur, S. Dolinar, M. Neifeld, M. J. Padgett, R. W. Boyd, J. H. Shapiro, and A. E. Willner, Opt. Lett. 38, 4062 (2013).

[27] J. Romero, D. Giovannini, S. Franke-Arnold, S. M. Barnett, and M. J. Padgett, Phys. Rev. A 86, 012334 (2012).

[28] A. Mair, A. Vaziri, G. Weihs, and A. Zeilinger, Nature 412, 313 (2001).

[29] H. He, M. E. J. Friese, N. R. Heckenberg, and H. RubinszteinDunlop, Phys. Rev. Lett. 75, 826 (1995).

[30] M. E. J. Friese, H. Rubinsztein-Dunlop, J. Gold, P. Hagberg, and D. Hanstorp, Appl. Phys. Lett. 78, 547 (2001).

[31] D. G. Grier, Nature 424, 810 (2003).

[32] T. A. Nieminen, J. Higuet, G. G. Knöner, V. L. Loke, S. Parkin, W. Singer, N. R. Heckenberg, and H. Rubinsztein-Dunlop, Proc. SPIE 6038, 603813 (2006).

[33] M. J. Padgett, Proc. SPIE 8637, 863702 (2013).

[34] N. B. Simpson, L. Allen, and M. J. Padgett, J. Mod. Opt. 43, 2485 (1996).

[35] N. B. Simpson, K. Dholakia, L. Allen, and M. J. Padgett, Opt. Lett. 22, 52 (1997).

[36] T. A. Nieminen, N. R. Heckenberg, and H. Rubinsztein-Dunlop, J. Mod. Opt. 48, 405 (2001).

[37] L. Chen, G. Zheng, and W. She, Phys. Rev. A 75, 061403 (2007).

[38] S. Furhapter, A. Jesacher, S. Bernet, and M. Ritsch-Marte, Opt. Express 13, 689 (2005).

[39] M. Chen, M. Mazilu, Y. Arita, E. M. Wright, and K. Dholakia, Opt. Lett. 38, 4919 (2013).

[40] M. D. Williams, M. M. Coles, K. Saadi, D. S. Bradshaw, and D. L. Andrews, Phys. Rev. Lett. 111, 153603 (2013).

[41] V. Y. Bazhenov, M. Vasnetsov, and M. Soskin, JETP Lett. 52, 429 (1990).

[42] V. Y. Bazhenov, M. Soskin, and M. Vasnetsov, J. Mod. Opt. 39, 985 (1992). 
[43] M. Mirhosseini, O. S. Magana-Loaiza, C. Chen, B. Rodenburg, M. Malik, and R. W. Boyd, Opt. Express 21, 30196 (2013).

[44] M. W. Beijersbergen, L. Allen, H. E. L. O. Vanderveen, and J. P. Woerdman, Opt. Commun. 96, 123 (1993).

[45] L. Marrucci, C. Manzo, and D. Paparo, Phys. Rev. Lett. 96, 163905 (2006).

[46] J. Sun, J. Zeng, and N. M. Litchinitser, Opt. Express 21, 14975 (2013).

[47] M. W. Beijersbergen, R. P. C. Coerwinkel, M. Kristensen, and J. P. Woerdman, Opt. Commun. 112, 321 (1994).

[48] N. R. Heckenberg, R. Mcduff, C. P. Smith, and A. G. White, Opt. Lett. 17, 221 (1992).

[49] A. S. Ostrovsky, C. Rickenstorff-Parrao, and V. Arrizón, Opt. Lett. 38, 534 (2013).

[50] L. Marrucci, J. Nanophotonics 7, 078598 (2013).

[51] C. Holbrow, E. Galvez, and M. Parks, Am. J. Phys. 70, 260 (2002).

[52] C. Maurer, A. Jesacher, S. Fürhapter, S. Bernet, and M. RitschMarte, New J. Phys. 9, 78 (2007).

[53] Z. Zhao, J. Wang, S. Li, and A. E. Willner, Opt. Lett. 38, 932 (2013).

[54] D. L. Andrews, M. M. Coles, M. D. Williams, and D. S. Bradshaw, Proc. SPIE 8813, 88130Y (2013).

[55] M. M. Coles, M. D. Williams, K. Saadi, D. S. Bradshaw, and D. L. Andrews, Laser Photonics Rev. 7, 1088 (2013).

[56] B. Bai, Y. Svirko, J. Turunen, and T. Vallius, Phys. Rev. A 76, 023811 (2007).

[57] V. A. Fedotov, A. S. Schwanecke, N. I. Zheludev, V. V. Khardikov, and S. L. Prosvirnin, Nano Lett. 7, 1996 (2007).

[58] E. Plum, X. X. Liu, V. A. Fedotov, Y. Chen, D. P. Tsai, and N. I. Zheludev, Phys. Rev. Lett. 102, 113902 (2009).

[59] N. A. Abdulrahman, Z. Fan, T. Tonooka, S. M. Kelly, N. Gadegaard, E. Hendry, A. O. Govorov, and M. Kadodwala, Nano Lett. 12, 977 (2012).

[60] N. Shitrit, S. Maayani, D. Veksler, V. Kleiner, and E. Hasman, Opt. Lett. 38, 4358 (2013).

[61] J. A. Salthouse and M. J. Ware, Point Group Character Tables and Related Data (Cambridge University Press, London, 1972).

[62] R. L. Carter, Molecular Symmetry and Group Theory (John Wiley, New York, 1998).

[63] L. Valkunas, D. Abramavicius, and T. Mancal, Molecular Excitation Dynamics and Relaxation: Quantum Theory and Spectroscopy (Wiley-VCH, Weinheim, 2013).

[64] E. W. Weisstein, CRC Concise Encyclopedia of Mathematics (Chapman \& Hall/CRC, Boca Raton, FL, 2003), p. 1077.

[65] R. D. Jenkins and D. L. Andrews, J. Chem. Phys. 118, 3470 (2003).

[66] D. S. Bradshaw and D. L. Andrews, J. Phys. Chem. A 117, 75 (2013).

[67] K. Robbie, M. J. Brett, and A. Lakhtakia, Nature 384, 616 (1996).

[68] T. G. Mackay and A. Lakhtakia, Opt. Express 15, 14689 (2007).

[69] Y. Zhu, F. Zhang, G. You, J. Liu, J. D. Zhang, A. Lakhtakia, and J. Xu, Appl. Phys. Express 5, 032102 (2012).

[70] H. Yu, M. Xu, Y. Zhao, Y. Wang, S. Han, H. Zhang, Z. Wang, and J. Wang, AIP Adv. 3, 092129 (2013).

[71] M. Zhi, K. Wang, X. Hua, H. Schuessler, J. Strohaber, and A. V. Sokolov, Opt. Express 21, 27750 (2013).
[72] G. Juzeliūnas, Chem. Phys. 198, 145 (1995).

[73] M. S. Soskin, V. N. Gorshkov, M. V. Vasnetsov, J. T. Malos, and N. R. Heckenberg, Phys. Rev. A 56, 4064 (1997).

[74] F. Ricci, W. Löffler, and M. P. van Exter, Opt. Express 20, 22961 (2012).

[75] M. R. Dennis and J. B. Götte, Phys. Rev. Lett. 109, 183903 (2012).

[76] N. Bozinovic, Y. Yue, Y. Ren, M. Tur, P. Kristensen, H. Huang, A. E. Willner, and S. Ramachandran, Science 340, 1545 (2013).

[77] D. L. Andrews, Phys. Rev. A 81, 033825 (2010).

[78] D. L. Andrews, Phys. Chem. Chem. Phys. 12, 7409 (2010).

[79] J. A. Rodrigo, T. Alieva, E. Abramochkin, and I. Castro, Opt. Express 21, 20544 (2013).

[80] B. Terhalle, T. Richter, A. S. Desyatnikov, D. N. Neshev, W. Krolikowski, F. Kaiser, C. Denz, and Y. S. Kivshar, Phys. Rev. Lett. 101, 013903 (2008).

[81] B. Terhalle, T. Richter, K. J. H. Law, D. Göries, P. Rose, T. J. Alexander, P. G. Kevrekidis, A. S. Desyatnikov, W. Krolikowski, F. Kaiser, C. Denz, and Y. S. Kivshar, Phys. Rev. A 79, 043821 (2009).

[82] F. Tamburini, E. Mari, A. Sponselli, B. Thidé, A. Bianchini, and R. Filippo, New J. Phys. 14, 033001 (2012).

[83] J. Brunel, O. Mongin, A. Jutand, I. Ledoux, J. Zyss, and M. Blanchard-Desce, Chem. Mater. 15, 4139 (2003).

[84] C. Rouxel, C. Le Droumaguet, Y. Macé, S. Clift, O. Mongin, E. Magnier, and M. Blanchard-Desce, Chem. Eur. J. 18, 12487 (2012).

[85] B. D. Gates, Q. B. Xu, M. Stewart, D. Ryan, C. G. Willson, and G. M. Whitesides, Chem. Rev. 105, 1171 (2005).

[86] J. V. Barth, G. Costantini, and K. Kern, Nature 437, 671 (2005).

[87] M. Knez, K. Nielsch, and L. Niinistö, Adv. Mater. 19, 3425 (2007).

[88] W. F. van Dorp and C. W. Hagen, J. Appl. Phys. 104, 081301 (2008).

[89] I. J. Hodgkinson, A. Lakhtakia, Q. H. Wu, L. De Silva, and M. W. McCall, Opt. Commun. 239, 353 (2004).

[90] A. Papakostas, A. Potts, D. M. Bagnall, S. L. Prosvirnin, H. J. Coles, and N. I. Zheludev, Phys. Rev. Lett. 90, 107404 (2003).

[91] E. Plum, J. Zhou, J. Dong, V. A. Fedotov, T. Koschny, C. M. Soukoulis, and N. I. Zheludev, Phys. Rev. B 79, 035407 (2009).

[92] F. Watt, A. A. Bettiol, J. A. Van Kan, E. J. Teo, and M. B. H. Breese, Int. J. Nanosci. 04, 269 (2005).

[93] L. J. Guo, Adv. Mater. 19, 495 (2007).

[94] Z. Xie, W. Yu, T. Wang, H. Zhang, Y. Fu, H. Liu, F. Li, Z. Lu, and Q. Sun, Plasmonics 6, 565 (2011).

[95] J. Abramson, M. Palma, S. J. Wind, and J. Hone, Adv. Mater. 24, 2207 (2012).

[96] A. Lundskog, C.-W. Hsu, K. Fredrik Karlsson, S. Amloy, D. Nilsson, U. Forsberg, P. Olof Holtz, and E. Janzen, Light Sci. Appl. 3, e139 (2014).

[97] A. F. Demirörs, P. P. Pillai, B. Kowalczyk, and B. A. Grzybowski, Nature 503, 99 (2013).

[98] X. Cai, J. Wang, M. J. Strain, B. Johnson-Morris, J. Zhu, M. Sorel, J. L. O'Brien, M. G. Thompson, and S. Yu, Science 338, 363 (2012).

[99] V. D’Ambrosio, E. Nagali, S. P. Walborn, L. Aolita, S. Slussarenko, L. Marrucci, and F. Sciarrino, Nat. Commun. 3, 961 (2012). 